\title{
The matching of a "one-dimensional" numerical simulation and experiment results for low viscosity Newtonian and non-Newtonian fluids during fast filament stretching and subsequent break-up
}

\author{
M. Tembely ${ }^{\mathrm{a})}$ \\ Laboratory for Geophysical and Industrial Flow Research (LEGI), UMR 5519, \\ University Joseph Fourier, Grenoble, BP 53, 38041 Grenoble Cedex, France \\ D. Vadillo and M. R. Mackley \\ Department of Chemical Engineering and Biotechnology, University of Cambridge, \\ Cambridge CB2 3RA, United Kingdom
}

\author{
A. Soucemarianadin \\ Laboratory for Geophysical and Industrial Flow Research (LEGI), UMR 5519, \\ University Joseph Fourier, Grenoble, BP 53, 38041 Grenoble Cedex, France
}

(Received 27 May 2011; final revision received 22 November 2011;

published 22 December 2011)

\begin{abstract}
Synopsis
This paper develops a model for fast filament stretching, thinning, and break-up for Newtonian and nonNewtonian fluids, and the results are compared against experimental data where fast filament relaxation occurs. A 1D approximation was coupled with the arbitrary Lagrangian Eulerian (ALE) formulation to perform simulations that captured both filament thinning and break-up. The modeling accounts for both the initial polymer stretching processes from the precise movement of the two moving pistons and also the subsequent thinning when the pistons are at rest. The simulations were first validated for a low viscosity Newtonian fluid matched to experimental data obtained from a recently developed apparatus, the Cambridge Trimaster. A non-Newtonian polymer fluid, with high frequency linear viscoelastic behavior characterized using a piezoaxial vibrator rheometer, was modeled using both an Oldroyd-B and FENECR single-mode constitutive models. The simulations of the filament deformation were compared with experiment. The simulations showed a generally reasonable agreement with both the stretch and subsequent relaxation experimental responses, although the mono mode models used in this paper were unable to capture all of the details for the experimental time evolution relaxation profile of the central filament diameter. (C) 2012 The Society of Rheology. [DOI: 10.1122/1.3669647]
\end{abstract}

\section{INTRODUCTION}

There are a number of situations where low viscosity fluids are stretched, form filaments and then break-up [Clasen et al. (2006b); Bhat et al. (2010)] and understanding

\footnotetext{
a) Author to whom correspondence should be addressed; electronic mail: moussa.tembely@ujf-grenoble.fr
} 
both the Newtonian and non-Newtonian fluid filament stretching and subsequent breakup into drops is of importance for optimizing processes such as ink jet printing, surface coating, or biological assays [Kuroiwa et al. (2003)]. In the past, fluid filament stretching studies have generally been restricted to mainly high viscosity fluids with the emphasis more on filament thinning behavior rather than break-up [see, for example, Matta and Tytus (1990); Yao and McKinley (1998); Orr and Sridhar (1996); Bach et al. (2002); McKinley and Sridhar (2002); Nielsen et al. (2006); Ma et al. (2008)]. Currently there is a very little literature describing the full stretching, thinning, and break-up situation for low viscosity dilute non-Newtonian fluids where the processes take place on the milliseconds time scale [Vadillo et al. (2010a)]. Short time thinning and break-up behavior are of particular relevance to ink jet processing where base viscosities are normally below $20 \mathrm{~m}$ Pas, and timescales are very short [Dong et al. (2006); Hoath et al. (2009)]. A review by McKinley and Sridhar (2002) covering both experimental and numerical aspects of filament thinning is particularly helpful in relation to previous work and physical insight. However, it should be noted that most previous works on viscoelastic fluids involve fluids with a high base velocity and relatively long relaxation times of say greater than $100 \mathrm{~ms}$. This paper is concerned with lower base viscosities and associated shorter relaxation times of less than $10 \mathrm{~ms}$.

It has been shown that $1 \mathrm{D}$ models have some potential to model accurately filament thinning for Newtonian as well as for polymeric fluids [Yildirim and Basaran (2001); Clasen et al. (2006b); Bhat et al. (2010)] but up till now the modeling results have seldom been compared with experimental stretching and thinning transient data for low viscosity fluids. Analytical and numerical works have mostly been devoted to following the time evolution of liquid bridge shapes, focusing on thread thinning, and early and late time asymptotics [Yildirim and Basaran (2001); Fontelos and Li (2004); Clasen et al. (2006a)]. Apart from [Clasen et al. (2006a)], these approaches do not offer direct comparison with experiments because of the absence of transients attached to the stretching process, i.e., the piston displacement history. However, [Wagner et al. (2005)] reported simulations where the detachment of a polymeric pendant drop has been used to analyze the dripping faucet phenomenon for non-Newtonian fluids. The comparisons between numerical and experimental results were quite good, although some properties such as the extensibility and the relaxation time had to be adjusted to fit the experimental results. Matallah et al. (2007) and Webster et al. (2008) have considered stretching transients in their work based on 2D axisymmetric models but do not have experimental comparisons to support their modeling. More recently, Castrejon-Pita et al. (2011) have reported achieving quantitative and qualitative agreements between experiment and 2D simulation, based on the Lagrangian-finite element method (FEM), of a driven (modulated or unmodulated) pressure liquid jet. It has been shown that the 1D approach can handle quite accurately 2D flow in some well defined cases: see, for example, the pioneering work by Eggers and Dupont (1994) and Eggers (1997). A direct comparison between 1D and 2D models may be found in the work of Yildirim and Basaran (2001) and more recently by Furlani and Hanchak (2011) and Hanchak (2009) where it has been demonstrated that the 1D model may capture the main features of drop formation although without no overturning reported. Finally, it is important to cite the work of Bhat et al. (2010) who explore the De-Oh region using a $1 \mathrm{D}$ model for identifying the region exhibiting the bead on structure formation where De and Oh are, respectively, the Deborah and Ohnesorge numbers. Bhat et al. (2010) use a one-dimensional analysis to cover a wide range of De and Oh values expeditiously and with quantitative accuracy similar to 2D models.

In view of the literature using one-dimensional analysis, a main objective of the present paper is to develop a model based on this analysis for use in extensional dominated 
flows such as those occurring in fluid stretching and compare the quality of the predictions with experimental data. The modeling incorporates both the initial high velocity stretching process of the pistons together with the subsequent filament thinning after stretch. These boundary conditions are particularly relevant to ink jet printing where a similar stretch and relaxation process occur when a drop exits the ink jet nozzle [Vadillo et al. (2010a); Morrison and Harlen (2010)]. An advantage of 1D modeling when compared with 2D modeling is a decrease in computing time of at least 2 orders of magnitude and this enables the lubrication model to effectively explore effects such as operating conditions and fluids properties [Hoeve et al. (2010)]. This benefit has also been put forward in other papers, [Ambravaneswaran et al. (2002)], [Bhat et al. (2010); Yildirim and Basaran (2001)]. In this paper, fluids were fully characterized using a high frequency rheometer [piezoaxial vibrator (PAV)] [Groß et al. (2002); Crassous et al. (2005); Kirschenmann (2003)] to obtain linear viscoelastic data of the fluids under test, and a specially designed filament stretching apparatus (Cambridge Trimaster MkII) was used to achieve controlled stretching [Vadillo et al. (2010a)]. On the numerical side, one-dimensional equations, taking full account of inertia, for both Newtonian and non-Newtonian models (Oldroyd-B, FENE-CR) were coupled with the arbitrary Lagrangian Eulerian (ALE) technique to describe carefully the movement of pistons of the filament stretching apparatus and the evolution of the filament boundary surface. The time dependence of piston displacement is of importance, especially for low viscosity fluids where filament stretching process must be achieved before inertia-capillary break-up occurs. This may require filament stretching rates as high as $\dot{\varepsilon}>100 s^{-1}$. The full description of the motion of the pistons also accounted for the acceleration and deceleration phases of the pistons, which are also of importance in defining the moving boundary conditions. The numerical results were compared with experimental data reported previously using the "Cambridge MkII Trimaster" filament stretcher [Vadillo et al. (2010a)].

The outline of the paper is as follows: in Sec. II, the mathematical formulation is detailed and the 1D constitutive equations derived. In addition, the fluids and the experimental apparatus are presented. The comparison of the model for fluid filament stretching and thinning is discussed in Sec. III together with results obtained on a Newtonian fluid as a first and necessary step. Section IV, summarizes the nonlinear Oldroyd-B and FENE-CR models and numerical simulations for the stretching and thinning of non-Newtonian fluids. Finally, conclusions are drawn in Sec. V.

\section{PROBLEM FORMULATION}

\section{A. 1D model governing equations}

The extensional flow of a liquid placed between two parallel coaxial moving pistons is considered (Fig. 1). Some details on the experimental procedures are given below but more details can be found in Vadillo et al. (2010a). The fluid is assumed to be either an incompressible Newtonian or non-Newtonian liquid. The kinematic viscosity of the solvent is $v_{\mathrm{s}}$ and the surface tension is $\sigma$, the fluid is rendered non-Newtonian through addition of small quantities of polymer into the solvent.

One way to deal with the Newtonian problem is to solve the full Navier-Stokes equation associated with robust schemes to track the interface deformation and break-up. Alternatively, a relevant one-dimensional approximation can be used that contains the main physics of the problem [Yildirim and Basaran (2001); Clasen et al. (2006a)]. The 1D equations of the problem have been described by Eggers and Dupont (1994), by expanding unknowns in a Taylor series in the radial coordinate for Newtonian fluids. The 


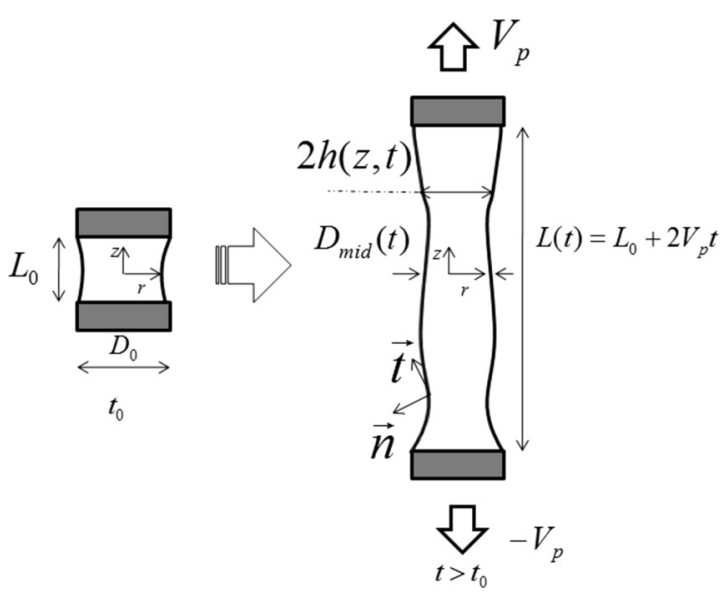

FIG. 1. Schematic of filament stretching and the coordinate system.

Navier-Stokes equations for an axisymmetric filament of velocity field $\vec{v} \equiv\left(v_{r}, v_{\theta}, v_{z}\right)$ and pressure $p$ are expressed as

Mass conservation

$$
\partial_{r} v_{r}+\partial_{z} v_{z}+v_{r} / r=0
$$

Momentum conservation

$$
\begin{aligned}
& \partial_{t} v_{r}+v_{r} \partial_{r} v_{r}+v_{z} \partial_{z} v_{r}=-\partial_{r} p+\nu_{s}\left(\partial_{r}^{2} v_{r}+\partial_{z}^{2} v_{r}+\partial_{r} v_{r} / r-v_{r} / r^{2}\right), \\
& \partial_{t} v_{z}+v_{r} \partial_{r} v_{z}+v_{z} \partial_{z} v_{z}=-\partial_{z} p+\nu_{s}\left(\partial_{r}^{2} v_{z}+\partial_{z}^{2} v_{z}+\partial_{r} v_{z} / r-v_{z} / r^{2}\right),
\end{aligned}
$$

where $\partial_{t}, \partial_{r}, \partial_{z}$ denote for the partial derivation with respect to $t, r$, and $z$, respectively.

The pressure and velocity field expansions in a power series in the radial direction $r$ results in

$$
\begin{aligned}
& p(r, z, t)=p_{0}(z, t)+p_{2}(z, t) r^{2}+\cdots, \\
& v_{z}(r, z, t)=v_{0}(z, t)+v_{2}(z, t) r^{2}+\cdots,
\end{aligned}
$$

Substituting Eq. (5) into Eq. (1), leads to

$$
v_{r}(r, z, t)=-v_{0}^{\prime}(z, t) \frac{r}{2}-v_{2}^{\prime}(z, t) \frac{r^{3}}{4}+\cdots .
$$

To close the problem, the following boundary conditions are used:

Kinematic boundary conditions

for the radius $h(z, t)$ of the jet

$$
\frac{d h}{d t}=\frac{\partial h}{\partial t}+v_{z} \frac{\partial h}{\partial z}=v_{r}(r=h, t) .
$$

Dynamic boundary conditions 
The dynamics boundary conditions at the liquid/air interface can be expressed through the normal and tangential stresses as detailed below.

- normal stress

The normal stress at the free surface is balanced by the curvature force associated with the surface tension

$$
\vec{n} \cdot \mathbf{T} \cdot \vec{n}=\sigma(\vec{\nabla} \cdot \vec{n})
$$

where $\vec{n}$ is the unit normal to the surface and the stress tensor $\mathbf{T}=-p \mathbf{I}+\eta_{s}\left[\vec{\nabla} \vec{v}+(\vec{\nabla} \vec{v})^{T}\right]$.

- tangential stress

The tangential stress at the free surface must balance the local surface tension gradient

$$
\vec{n} \cdot \mathbf{T} \cdot \vec{t}=(\vec{\nabla} \sigma) \cdot \vec{t}=0,
$$

assuming a constant surface tension $\sigma$ and $\vec{t}$ being the unit tangent to the interface.

Finally, no-slip conditions are imposed at the piston surfaces

$$
\begin{gathered}
h(z=-L / 2, t)=h(z=L / 2, t)=D_{0} / 2, \\
v(z=-L / 2, t)=-V_{p}, v(z=L / 2, t)=V_{p},
\end{gathered}
$$

where $D_{0}$ and $V_{p}$ are the piston diameter and velocity, respectively.

Replacing the radial expansions (4)-(6) into Eqs. (1) and (2) and taking the lower order results in $r$ lead to the nonlinear one-dimensional equations describing the filament dynamics

$$
\partial_{t} h+v h^{\prime}+v^{\prime} h / 2=0
$$

and

$$
\partial_{t} v+v v^{\prime}=-\frac{\sigma \kappa^{\prime}}{\rho}+3 \nu_{s} \frac{\left(v^{\prime} h^{2}\right)^{\prime}}{h^{2}},
$$

where prime $\left({ }^{\prime}\right)$ denotes the derivative with respect to $z$ coordinates.

To avoid instability in the solution and have the complete ability to represent a rounded drop, the full expression of the curvature given in Eq. (14) is taken. This is not asymptotically correct as shown in Papageorgiou (1995), but this choice has been justified and used by many authors [Yildirim and Basaran (2001); Fontelos and Li (2004); Clasen et al. (2006a); Bhat et al. (2010)]. The radial expansion requires replacing the mean curvature by the leading-order expression $1 / h$ alone, but the applicability of the equations is improved by accounting for the full curvature, since the equations retrieve a spherical drop among its equilibrium solutions

$$
\kappa=\frac{1}{h\left(1+h^{\prime 2}\right)^{1 / 2}}-\frac{h^{\prime \prime}}{\left(1+h^{\prime 2}\right)^{3 / 2}} .
$$

Eqs. (12) and (13) have been shown to give a good approximation to the full Navier-Stokes solution [Eggers and Dupont (1994)]. 
The contribution of a non-Newtonian polymer leads to an extra-stress tensor $\left(\sigma_{z z}-\sigma_{r r}\right)$, and the momentum conservation equations becomes in dimensionless form

$$
\partial_{t} v+v v^{\prime}=-\kappa^{\prime}+3 \tilde{\nu}_{s} \frac{\left(v^{\prime} h^{2}\right)^{\prime}}{h^{2}}+\frac{1}{h^{2}}\left[h^{2}\left(\sigma_{z z}-\sigma_{r r}\right)\right]^{\prime},
$$

using $\tau$ as the characteristic inertio-capillary time scale of the system defined by $\tau=\sqrt{\rho x_{0}^{3} / \sigma}$, and $x_{0}$ as a unit of length, $\tilde{v}_{s}=v_{s} \tau / x_{0}^{2}$.

The extra-stress depends on the non-Newtonian model chosen and the resulting equations will be detailed later.

According to the findings of Yao and McKinley (1998) stress boundary layers are usually present in the case of low initial aspect ratio experiments. In our work, the fluid is lowly viscoelastic, and the initial aspect ratio is $L_{0} / R_{0}=1$. Following [Clasen et al. (2006a)], we may disregard the radial variation in the axial polymeric stress, which, however, may be introduced easily in the 1D formulation [Kolte and Szabo (1999)].

\section{B. Numerical modeling}

The governing equations were solved using COMSOL, a commercial FEM code. The ALE technique was used to handle the dynamics of the boundaries with a moving grid where the new mesh coordinates are computed based on the motion of the boundaries of the structure. The governing equations are solved using these moving coordinates. The model, as presented here, has the additional advantage of not needing any unphysical artificial viscosity just for the purpose of pinning the fluid at the piston as required in Clasen et al. (2006a).

\section{Experimental setup}

The experimental setup, described in Vadillo et al. (2010a), is a fast filament stretching and thinning, extensional rheometer, the so-called "Cambridge Trimaster." This apparatus performs filament stretching at a near constant velocity for a fluid initially placed between two pistons of initial diameter $D_{0}$ of $1.2 \mathrm{~mm}$. Both pistons are attached on the opposite sides of a belt and move symmetrically apart for a given distance allowing the midfilament to remain in a central position during the experiment (Fig. 1).

The pistons can move from a distance of $10 \mu \mathrm{m}$ to $10 \mathrm{~cm}$ at a maximum relative velocity of $1 \mathrm{~m} / \mathrm{s}$. When the pistons stop, the filament self-thins under the action of the capillary and viscous forces. The pistons size is selected to obtain small Bond numbers (Bo $=\rho g D_{0}^{2} / 4 \sigma=0.1$, where $\rho, \sigma$, g, are the fluid density, surface tension, and gravitational constant, respectively), so that gravitational effects were negligible in comparison to capillary forces. A high speed camera (Photron Fastcam 1024 PCI) is coupled with the Cambridge Trimaster, allowing transient profiles to be recorded at a frame rate as fast as 100000 frames per second at a reduced resolution of $32 \times 32$ pixels and with a shutter time as low as $3 \mu \mathrm{s}$. The pixel size is close to $5.5 \mu \mathrm{m}$, and the illumination used was a continuous light guided by fibre optics. The high speed pictures are recorded at $6000 \mathrm{fps}$, for a picture size of $128 \times 256$ pixels with a shutter time of $3 \mu$ s. The filament thinning measurement, as well as the filament break-up behavior, is obtained using automatic image processing specifically developed for, and included within, the Cambridge Trimaster software package. This apparatus enabled the measurement of the transient elongational viscosity using the midfilament evolution as well as the observation of fluid filament profiles. The experimental filament stretching conditions used in this paper, unless stated otherwise, are an 
initial gap size $L_{0}$ of $0.6 \mathrm{~mm}$ and a relative piston speed $V_{p}$ of $150 \mathrm{~mm} / \mathrm{s}$ corresponding to a filament strain rate $V_{p} / L_{0}=250 \mathrm{~s}^{-1}$. The stretching distance may be modified, and displacements of $0.8 \mathrm{~mm}$ and $1.6 \mathrm{~mm}$ are investigated in the present work.

The PAV is a dynamic squeeze flow rheometer used to characterize linear viscoelasticity (LVE) of soft matter fluids such as low viscosity polymeric solutions. This apparatus that has been detailed in Groß et al. (2002), Kirschenmann (2003), Crassous et al. (2005), and Vadillo et al. (2010b) and is capable of probing fluids with viscosity as low as $1 \mathrm{mPa}$ s over a range of frequencies between $0.1 \mathrm{~Hz}$ and $10000 \mathrm{~Hz}$. The PAV measures the complex modulus $G^{*}$ of the test fluid with $G^{*}=G^{\prime}+i G^{\prime \prime}$ and where $G^{\prime}$ is the storage modulus and $\mathrm{G}^{\prime \prime}$ is the loss modulus. The complex viscosity $\eta^{*}$ is related to the complex modulus by $\eta^{*}=\mathrm{G}^{*} / \omega$, where $\omega$ is the angular frequency. The viscosities of the two different fluids are determined from PAV low frequency complex viscosity data.

Diethyl phthalate (DEP) was chosen as the Newtonian fluid, and the second fluid was a non-Newtonian mixture of monodisperse polystyrene $110000 \mathrm{~kg} / \mathrm{kmol}$ (PS110) dissolved at 2.5 wt. \% in DEP. The critical polymer overlap concentration $c^{*}$ was determined using $c^{*}=0.77 /[\eta]$, where $[\eta]$ is intrinsic viscosity [Graessley (1980)]. Using the Mark-Houwink-Sakurada equation $[\eta]=\mathrm{K}_{[\eta]} \mathrm{M}^{\gamma}$, where $K_{[\eta]}=8.1 \times 10^{-3}$ and $\gamma=0.704$, a $c^{*}$ of $2.40 \%$ is obtained for $110000 \mathrm{~g} / \mathrm{mol}$ polystyrene for DEP-PS solution [Clasen et al. (2006b)]. Finally, fluid surface tension was measured using a "Wilhelmy plate" method and a "SITA, proline t15" bubble pressure tensiometer, and a typical value of $37 \mathrm{mN} / \mathrm{m}$ was obtained for both the pure DEP and the mixture of $2.5 \mathrm{wt}$. \% PS110 in DEP. Fluids physical properties are summarized in Table I.

\section{NEWTONIAN LIQUID FILAMENT STRETCHING AND THINNING}

In order to achieve a realistic match between simulation and experimental results it was necessary to accurately introduce the initial piston movement boundary conditions. Acceleration and deceleration times to reach the setup velocity as well as subsequent damping oscillations, may usually be neglected for the case of high viscosity or highly viscoelastic fluids, as the stretching time $\delta \mathrm{t}_{0}$ is a very short duration \{typically $\sim 50 \mathrm{~ms}$ [Rodd et al. (2005)]\} compared to thinning and break-up times (>100 ms) [see, for example, Liang and Mackley (1994); Anna and McKinley (2001); Rodd et al. (2005)]. However, in the case of filament stretching and thinning of low viscosity and/or low viscoelastic fluids, such as the ones described in this paper, to ensure that the piston stop before the capillary break-up, $\delta \mathrm{t}_{0}$ should be shorter than the inertio-capillary time $t_{i}\left\{t_{i}=1.95 \sqrt{\rho R_{0}^{3} / \sigma}=1.95 \tau\right.$ [Rodd et al. (2005)] $\}$. This time has been estimated at $5 \mathrm{~ms}$ in the case of DEP indicating that a filament stretch strain rate of $200 \mathrm{~s}^{-1}$ is required. In such cases, the initial piston movement must be taken into account in the simulation. Using high speed camera data, the experimental displacement of Trimaster pistons with time was recorded and is shown in Fig. 2 where the acceleration phase between 2.4 and 4 $\mathrm{ms}$, the constant velocity phase between 4 and $6.4 \mathrm{~ms}$ (corresponding to a linear evolution of the piston position) and the deceleration phase between 6.4 and $8 \mathrm{~ms}$, can be observed. A mathematical function of the form $D_{p}(t)=a \tanh [b(t-c)]+d$, with $a=245 \mu \mathrm{m}$,

TABLE I. Physical properties of the fluids, viscosity came from PAV low frequency complex viscosity data.

\begin{tabular}{lccc}
\hline \hline Fluids & Surface tension $(\sigma)\left(\mathrm{mN} \mathrm{m}^{-1}\right)$ & Viscosity $\left(\eta_{0}\right)(\mathrm{mPa} \mathrm{s})$ & Density $(\rho)\left(\mathrm{kg} \mathrm{m}^{-3}\right)$ \\
\hline DEP & 37 & 14 & 1120 \\
DEP $+2.5 \%$ PS & 37 & 32 & 1120 \\
\hline \hline
\end{tabular}


$b=0.6 \mathrm{~ms}^{-1}, c=5.4 \mathrm{~ms}$, and $d=510 \mu \mathrm{m}$, was used to fit the displacement of the pistons and a best fit is also shown in Fig. 2. The instantaneous velocity of the pistons, $V_{p}(t)=a b / \cosh ^{2}[b(t-c)]$, that corresponds to the derivative of $D_{p}(t)$ can subsequently be used in the numerical simulation as boundary conditions for the displacement of the piston.

\section{A. Midfilament evolution}

In filament stretching and filament thinning experiments, the midfilament evolution $D_{\text {mid }}(t)$ is often used to describe the fluid behavior [see, for example, Liang and Mackley (1994); Anna and McKinley (2001); Rodd et al. (2005)]. To further emphasise the importance of correctly accounting for the "real" motion of the pistons for the simulation of fast breaking fluids, two simulations using a constant piston speed of $V_{p}=75 \mathrm{~mm} / \mathrm{s}$ and $V_{p}=150 \mathrm{~mm} / \mathrm{s}$, as well as $V_{p}(t)$ obtained from derivative of the best fit of $D_{p}(t)$, have been tested, and the results are shown in Fig. 3. Comparison of the simulation shows several significant differences. At the early stage of the stretching process, the experimental piston velocity boundary conditions $V_{p}(t)$ lead to a slower thinning of the midfilament. When the pistons have reached a stable velocity, the midfilament is found to thin in a manner which is apparently linear for all three boundaries' conditions. However, due to an average experimental velocity higher than $V_{p}=75 \mathrm{~mm} / \mathrm{s}$, simulation results obtained with $V_{p}(t)$ cross over with the one obtained with $V_{p}=75 \mathrm{~mm} / \mathrm{s}$. Subsequently, the simulated midfilament was found to be thinner for $V_{p}=150 \mathrm{~mm} / \mathrm{s}$ and thicker for $V_{p}=75$ $\mathrm{mm} / \mathrm{s}$ when the pistons stop. In the filament, visco-capillary thinning part, $D_{\text {mid }}(t)$ decreases as documented in the literature for Newtonian fluids [Bazilevsky et al. (1990); Liang and Mackley (1994); Entov and Hinch (1997); Kolte and Szabo (1999); Vadillo et al. (2010a)]. The break-up time was found to be the shortest for $V_{p}=150 \mathrm{~mm} / \mathrm{s}$, the longest for $V_{p}=75 \mathrm{~mm} / \mathrm{s}$ and to perfectly match the experimental break-up for $V_{p}(t)$ (Fig. 4). It can also be noticed than using $V_{p}=150 \mathrm{~mm} / \mathrm{s}$ leads to a break-up time that is comparable with $t_{i}$ and the stretching time. Nevertheless, despite the clear differences visible in the midfilament evolution, no significant effect of the boundary conditions is observed on the break-up transient profile.

The results of simulations of the evolution of the midfilament, using the best fit of the experimental dynamic of the pistons as boundary conditions, are compared with the experimental data (Fig. 4) in the case of the Newtonian DEP and excellent agreement was obtained throughout the entire stretching and thinning process. Both the viscocapillary thinning of $D_{\text {mid }}$ as well as the break-up time were correctly predicted. The

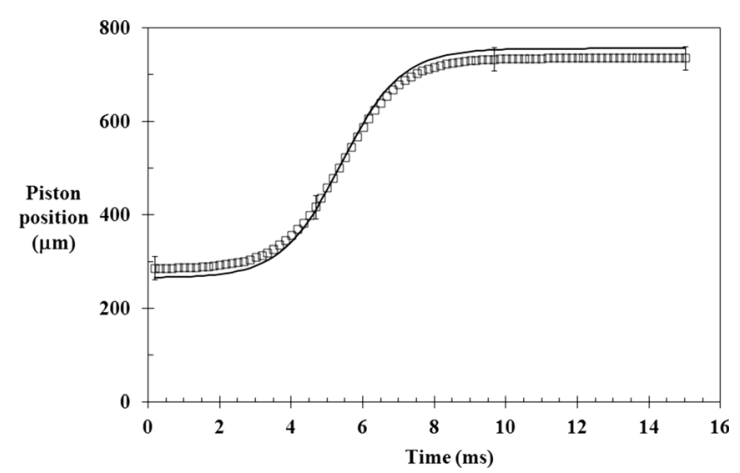

FIG. 2. Comparison between ( $\square$ ) experimental and (-) the model fit given in Sec. III of the piston motion as a function of time. 


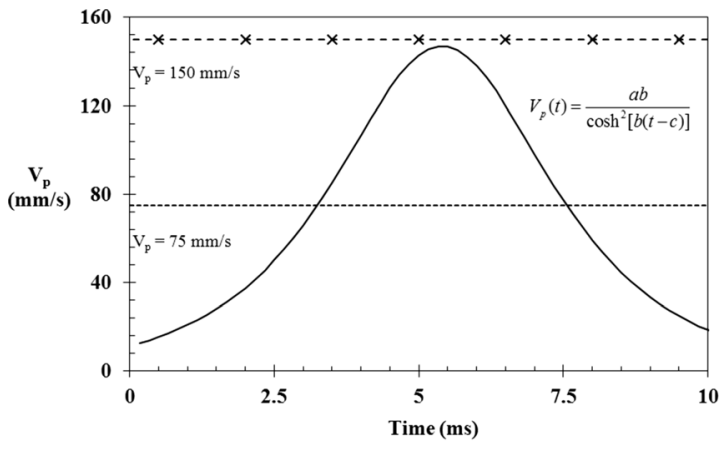

a

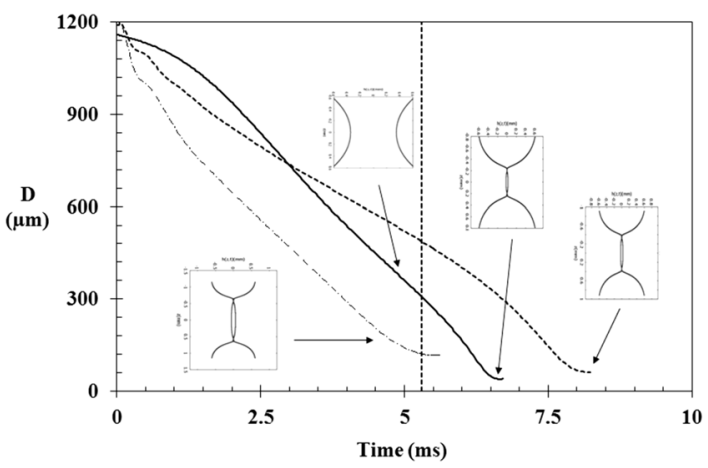

FIG. 3. (a) Plot of the piston velocity evolution (---) $75 \mathrm{~mm} / \mathrm{s},(\mathbf{x}--\mathbf{x}) 150 \mathrm{~mm} / \mathrm{s}$, (-) best fit of the experimental piston velocity $V_{p}(t)$ and (b) the corresponding numerical midfilament evolution for Newtonian DEP using the three piston velocity boundary conditions; (-- $) 150 \mathrm{~mm} / \mathrm{s},(--) 75 \mathrm{~mm} / \mathrm{s}$, and (-) best fit of the experimental piston velocity $V_{p}(\mathrm{t})$. (---) Corresponds to piston cessation of motion.

dominant mechanism is the piston motion and when it stops all forces (viscosity, capillarity, and inertia) take over until break-up. Instead of $D_{\text {mid }}$, the midfilament diameter preferentially used in rheology, [McKinley and Sridhar (2002), one may check the autosimilar solution by focusing on $D_{\min }$. For that purpose, as detailed in Eggers (1997), an autosimilar behavior may be recovered by using the following intrinsic length $l_{\mathrm{v}}=\nu_{\mathrm{s}}^{2} \rho / \sigma$

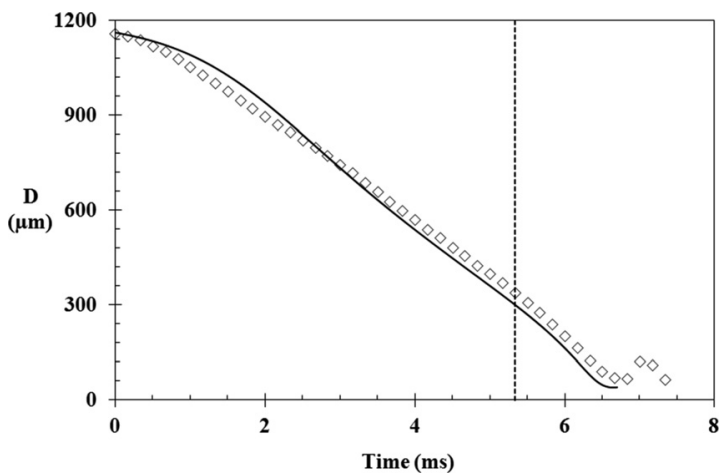

FIG. 4. Plot of midfilament diameter as a function of time. (-) Numerical midfilament evolution compared to $(\diamond)$ experimental measurement for Newtonian DEP, (---) corresponds to piston cessation of motion (aspect ratio 2.3). Initial gap size: $0.6 \mathrm{~mm}$, final gap size: $1.4 \mathrm{~mm}$, pistons relative velocity: $150 \mathrm{~mm} / \mathrm{s}$. 
and timescales $t_{\mathrm{v}}=\nu_{\mathrm{s}}^{3} \rho^{2} / \sigma^{2}$. Under these conditions, viscous capillarity-driven pinch-off is described by $D_{\min }(t) \approx 0.14\left(t_{\mathrm{b}}-t\right)$, whereas if inertia is important, the corresponding law is $D_{\min }(t) \approx 0.06\left(t_{\mathrm{b}}-t\right)$, where $t_{\mathrm{b}}$ is the dimensionless breakup-time and $t$ is the actual dimensionless time. Our simulations show that for $\mathrm{Oh}=\left(l_{\mathrm{v}} / R_{0}\right)^{1 / 2}=0.08$, the minimum neck diameter $D_{\min }$ is comprised between a viscous-capillary regime and an inertio-capillary one (Fig. 5). We have also plotted the experimental points, which are in good agreement with the simulations. One may note that the evolution of $D_{\text {mid }}$, near breakup (fluid build up) is in sharp contrast with the behavior of $D_{\min }$ (necking at ends). The current 1D model may be extended to deal with the case when the filament breaks up as shown in Eggers (1997) or even to model subsequent coalescence of two fluid volumes [Furlani and Hanchak (2011)]. However, overturning which occurs for low viscosity fluids cannot be represented.

Such possibilities of the 1D model have not been attempted in this work, and we limit the calculations to break-up which is said to have occurred when the dimensionless minimum filament radius $h_{\mathrm{min}} / R_{0}$ drops below $10^{-4}$ corresponding, in other words, to $h_{\min }<0.06 \mu \mathrm{m}$. In the following, the operating conditions derived from the experimental piston motion will systematically be used.

\section{B. Transient profiles}

A comparison between the experimental and the predicted transient profiles is shown in Figs. 6 and 7 for the Newtonian DEP fluid. The simulated transient profiles and the experimentally captured ones are in good agreement and in particular, the prediction of the top and bottom pinch off which eventually leads to the formation of a central droplet is accurately simulated. This demonstrates that the 1D model, also called "slender filament approximation," remains valid even for an aspect ratio close to 1, highlighting the approximations effectiveness. The average computational time for 1D simulation is shorter than $5 \mathrm{~min}$, whatever the type of fluid considered, using a Pentium Dual Core laptop computer with 4 GB RAM. This computational time is significantly shorter than for a full 2D simulation.

It was observed that the top and bottom pinch off behavior observed for the low viscosity fluids reported in this paper are different to the cases for higher viscosity Newtonian fluids reported in Liang and Mackley (1994) where the central filament decayed without end pinching. The transition between top and bottom pinch off and "central"

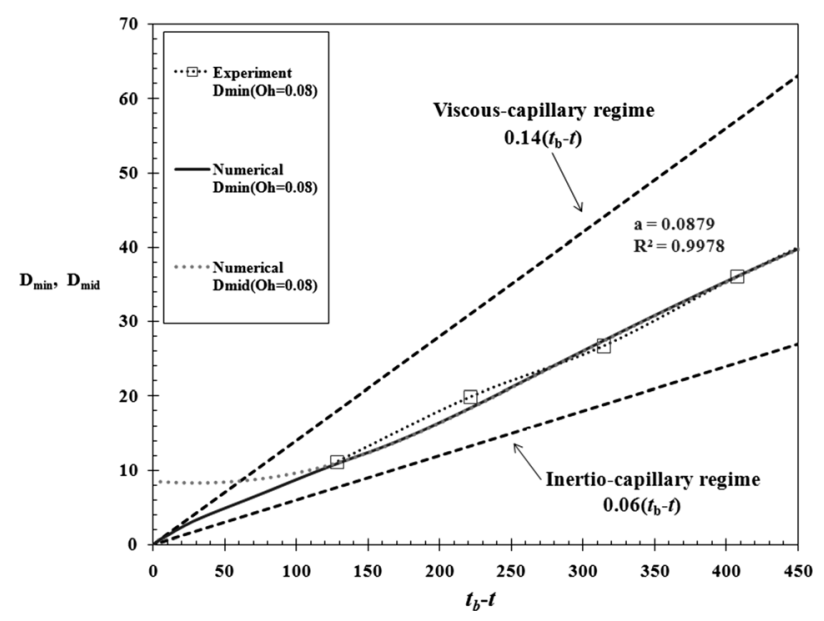

FIG. 5. Behavior of minimum and midfilament diameter close to break-up. The slope of the linear best fit for $\mathrm{D}_{\text {min }}$ calculated numerically is $a=0.0879$ with a coefficient of determination $R^{2}=0.9978$. 

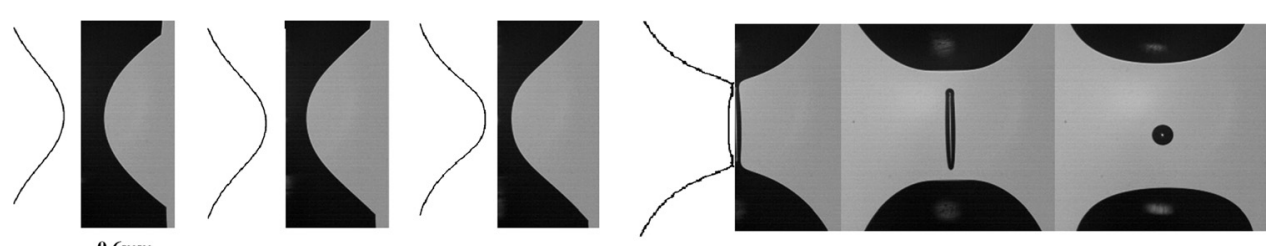

FIG. 6. Comparison between (right) numerical profiles and (left) the corresponding experimental profiles at different specified times in the case of DEP. Initial gap size: $0.6 \mathrm{~mm}$, final gap size: $1.4 \mathrm{~mm}$, pistons relative velocity: $150 \mathrm{~mm} / \mathrm{s}$. Times are, from left to right, $5.3 \mathrm{~ms}, 5.8 \mathrm{~ms}, 6.7 \mathrm{~ms}, 7 \mathrm{~ms}, 7.2 \mathrm{~ms}$, and $7.7 \mathrm{~ms}$.

filament break-up corresponds to the transition between inertio-capillary break-up, where the filament thins as an inviscid liquid, and viscous break-up. Such behavior may also be observed for the dilute polymer case to be detailed in Sec. IV despite the stabilization of the filament induced by the presence of the polymer.

\section{NON-NEWTONIAN LIQUID FILAMENT STRETCHING AND THINNING}

\section{A. Single-mode modeling}

In this section, the modeling of the behavior of the polymer solution $(\mathrm{DEP}+2.5 \%$ PS110) is considered, assuming the fluid to be an incompressible viscoelastic fluid modeled by a single-mode Oldroyd-B or FENE-CR model. This approach was motivated by the fact that it has been established that, in the filament stretching experiment, the dynamics of the elasto-capillary thinning was controlled by the longest relaxation time [Entov and Hinch (1997); Anna and McKinley (2001)]. Moreover, it has been demonstrated that high frequency linear viscoelastic measurements of solution DEP $+2.5 \mathrm{wt}$ \% of PS110 behaved as a singlemode Maxwell fluid with $\mathrm{G}^{\prime \prime}$ and $\mathrm{G}^{\prime}$ increasing with a slope of one and two with frequency, respectively [Vadillo et al. (2010b)]. Although it is not clear at this point which route is the most appropriate, a single-mode description was tested to evaluate its performance.

In order to establish the non-Newtonian 1D model description, the previous Eqs. (10)-(12), and (15) are completed by the addition of extra-stresses $\sigma_{z z}-\sigma_{r r}$ due to the polymer. The two different non-Newtonian constitutive equations are as follows:

- Oldroyd-B

For this model the extra-stresses satisfy

$$
\begin{gathered}
\frac{\partial \sigma_{z z}}{\partial t}+v \frac{\partial \sigma_{z z}}{\partial z}+\left(\frac{1}{D_{e}}-2 \frac{\partial v}{\partial z}\right) \sigma_{z z}=2 \frac{\tilde{\nu}_{p}}{D_{e}} \frac{\partial v}{\partial z} \\
\frac{\partial \sigma_{r r}}{\partial t}+v \frac{\partial \sigma_{r r}}{\partial z}+\left(\frac{1}{D_{e}}+\frac{\partial v}{\partial z}\right) \sigma_{r r}=-\frac{\tilde{\nu}_{p}}{D_{e}} \frac{\partial v}{\partial z}
\end{gathered}
$$

where $D_{e}=\lambda / \tau$ is the Deborah number, with $\lambda$ the relaxation time of the polymer. $\tilde{v}_{p}=v_{p} \tau / x_{0}^{2}$ and $v_{p}$ represents the contribution of the polymer to the viscosity [Clasen et al. (2006a)].

\section{- FENE-CR}

In this approach, the polymer contribution is described by a finitely extensible nonlinear elastic (FENE) dumbbell model which makes use of the conformation tensor $\mathbf{A}$, and the stress tensor reads [Chilcott and Rallison (1988)] 


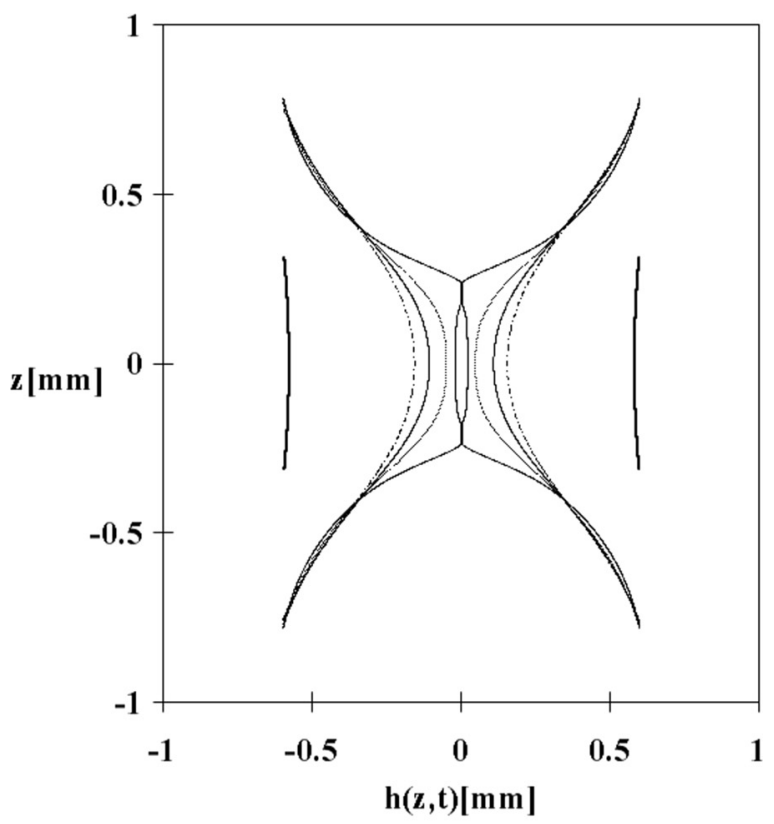

a

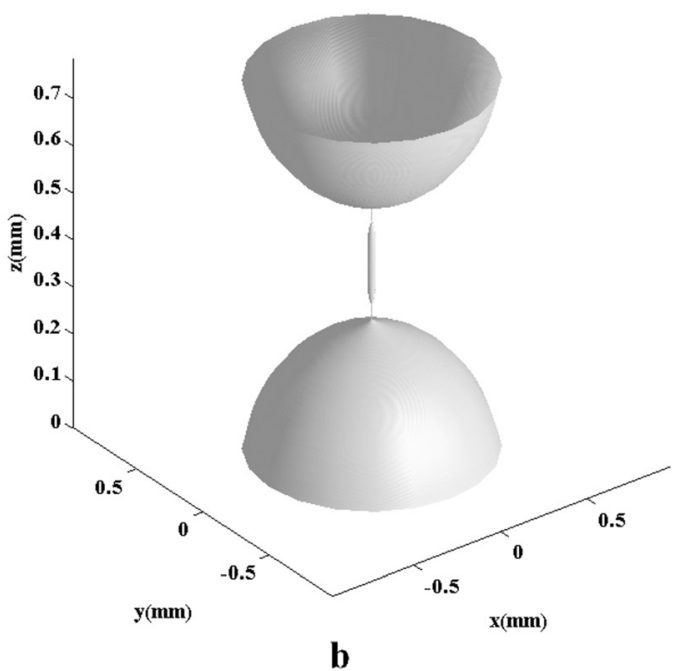

FIG. 7. (a) Numerical simulation of transient profiles of filament break-up in the case of DEP (Newtonian) at different specified times. (-) $0 \mathrm{~ms},(-\cdot) 5.3 \mathrm{~ms},(-) 5.8 \mathrm{~ms},(\ldots) 6.3 \mathrm{~ms}$, and (-) $6.7 \mathrm{~ms}$. (b) 3D representation of the filament at the point of break-up. Initial gap size: $0.6 \mathrm{~mm}$, final gap size: $1.4 \mathrm{~mm}$, pistons relative velocity: $150 \mathrm{~mm} / \mathrm{s}$.

$$
\boldsymbol{\sigma}=G f(R)(\mathbf{A}-\mathbf{I}),
$$

where $G$ is the elastic modulus, $f(R)$ is the finite extensibility factor related to the finite extensibility parameter $L$, representing the ratio of a fully extended polymer (dumbbell) to its equilibrium length. $L$ can be described in terms of molecular parameters as 


$$
L=\sqrt{3}\left[\frac{j(\sin \theta / 2)^{2} M_{w}}{C_{\infty} M_{u}}\right]^{1-\nu},
$$

where $\theta$ is C-C bond angle, $j$ is the number of bonds of a monomer of molar mass $M_{u}$, $C_{\infty}$ is the characteristic ratio for a given polymer, $M_{w}$ is the molecular weight of the polymer and $\nu$ is the excluded volume exponent [Clasen et al. (2006b)]. For the one-dimensional model in dimensionless form, it leads to

$$
\begin{gathered}
R=\operatorname{tr}(\mathbf{A})=A_{z z}+2 A_{r r}, \\
\sigma_{z z}=\tilde{G} f(R)\left(A_{z z}-1\right), \quad \sigma_{r r}=\tilde{G} f(R)\left(A_{r r}-1\right),
\end{gathered}
$$

where $\tilde{G}=\tilde{\nu}_{p} / D_{e}$.

$$
f(R)=\frac{1}{1-R / L^{2}}
$$

The evolution equations for the conformation tensor A may be written as follows:

$$
\begin{gathered}
\frac{\partial A_{r r}}{\partial t}+v \frac{\partial A_{r r}}{\partial z}+\left(\frac{f(R)}{D_{e}}+\frac{\partial v}{\partial z}\right) A_{r r}=\frac{f(R)}{D_{e}}, \\
\frac{\partial A_{z z}}{\partial t}+v \frac{\partial A_{z z}}{\partial z}+\left(\frac{f(R)}{D_{e}}-2 \frac{\partial v}{\partial z}\right) A_{z z}=\frac{f(R)}{D_{e}} .
\end{gathered}
$$

The constitutive equations require values for the fluid relaxation time $\lambda$, shear modulus $g$ and polymer extensibility $\mathrm{L}$ as input parameters. The problem reduces to the determination of $\lambda$ and g, after taking into account that the complex viscosity of the polymer fluid $\eta^{*}$ and solvent viscosity (DEP) $\eta_{s}$ are obtained from the PAV low frequency measurements (Table I). The fluid complex viscosity can be expressed, in a mono mode configuration, by

$$
\left|\eta^{*}\right|=\eta_{\mathrm{s}}+\lambda \cdot \mathrm{g} .
$$

From this point in the paper, two different routes were followed to determine the rheological parameters. The first route consisted of using the experimental PAV linear viscoelastic data and best fitting to a mono mode Maxwell behavior. The elastic and viscous modulus expressed in the mono mode approach can be expressed as

$$
\begin{gathered}
G^{\prime}(\omega)=\sum_{i=1}^{N_{\text {modes }}}\left(\frac{g_{i}\left(\lambda_{i} \omega\right)^{2}}{1+\left(\lambda_{i} \omega\right)^{2}}\right) \stackrel{i=1}{\sim} \frac{\mathrm{g} \cdot(\omega \cdot \lambda)^{2}}{1+(\omega \cdot \lambda)^{2}}, \\
G^{\prime \prime}(\omega)=\eta_{s} \omega+\sum_{i=1}^{N_{\text {modes }}}\left(\frac{g_{i} \lambda_{i} \omega}{1+\left(\lambda_{i} \omega\right)^{2}}\right) \stackrel{i=1}{\sim} \eta_{\mathrm{s}} \cdot \omega+\frac{\lambda \cdot \mathrm{g} \cdot \omega}{1+(\omega \cdot \lambda)^{2}},
\end{gathered}
$$

where $\omega$ is the frequency in $\mathrm{rad} / \mathrm{s}$ and $\left|\eta^{*}\right|=\frac{\sqrt{G^{\prime 2}+G^{\prime \prime 2}}}{\omega}$. It should be noted here that the loss modulus description chosen includes both the solvent and polymer viscous 
contribution. Rheological parameters are embedded in the experimentally determined coefficient and, a single-mode description only allows a unique couple $(\lambda, \mathrm{g})$ that satisfies the equations. Best fit of single-mode Maxwell model was obtained with $\lambda=8 \mu$ s and $g=2469.3 \mathrm{~Pa}$ [Fig. 8(a)]. It was noticed that the relaxation time obtained from the experimental PAV data is of the same order of magnitude as the longest Zimm relaxation time. This model is the one preferentially used in the literature to fit LVE data although the solution characterized is often far from their dilute regime [see, for example, Anna and McKinley (2001); Clasen et al. (2006b)]. The longest "Zimm relaxation time" $\lambda_{\mathrm{z}}$ can be expressed as follows [Clasen et al. (2006b)]:

$$
\lambda_{z}=\frac{1}{U_{\eta \lambda}} \frac{[\eta] \eta_{s} M_{w}}{R T}
$$

where $U_{\eta \lambda}$ is the universal ratio of a characteristic relaxation time $\lambda_{\mathrm{n}}$ of a dilute polymer system and estimated around 1.8 for a good solvent, $[\eta]$ is the intrinsic viscosity obtained from LVE data, $\eta_{s}$ is the solvent viscosity, $R=8.314 \mathrm{~J} / \mathrm{mol} \mathrm{K}$ is the universal gas constant, $T$ is the absolute temperature in $\mathrm{K}$. In the present case, $\lambda_{z}$ of order of $16.6 \mu \mathrm{s}$ has been estimated.

A second set of Maxwell parameters was derived from experimental filament thinning data. It has been previously reported that fluid relaxation can be significantly larger in extensional deformations when compared to simple shear flow [Clasen et al. (2006b)]. Experimentally, the elastic nature of the fluid is described, during thinning of the midfilament diameter, following an exponential law of the form [McKinley and Sridhar (2002); Tuladhar and Mackley (2008)]:

$$
D_{\text {mid }}(t) \sim \exp \left(-\frac{t}{3 . \lambda}\right)
$$

In the case of DEP +2.5 wt. \% of PS110, the fit of the Cambridge Trimaster experimental exponential filament decay of the midfilament leads to a longest extensional relaxation time $\lambda_{\text {ext }} \sim 1.19 \mathrm{~ms}$, or $D e=\lambda_{\text {ext }} / \tau \sim 0.47$ where $\tau=\sqrt{\rho R_{0}^{3} / \sigma}$. This extensional relaxation time is significantly larger than the relaxation time extracted from LVE data which is a result consistent with previously published finding reported by Clasen et al. (2006b). The shear modulus $g$ for the Maxwell model was obtained from knowing the complex modulus of the fluid and solvent and again using Eq. (25) [Fig. 8(b)].

Figure 8(c) shows a comparison plot of PAV complex viscosity as a function of frequency for the experimental fluid tested and the two Maxwell models tested. In both model cases, the low frequency match is good; however, the long time filament thinning relaxation time starts to shear thin at a lower frequency than the PAV short relaxation time data. The high frequency plateau of the filament thinning timescale is a consequence of the Newtonian solvent contribution.

Two separate simulations, using $(\lambda, \mathrm{g})$ couples extracted from both (i) experimental PAV, LVE, data and (ii) experimental extensional relaxation data have been used. The simulation parameters used were, namely, (i) $\lambda_{\mathrm{lve}} \sim 8 \mu$ s (or $D e=0.00145$ ) and $g=2469.3 \mathrm{~Pa}$ and (ii) $\lambda_{\text {ext }} \sim 1.19 \mathrm{~ms}(D e \sim 0.47), g=15 \mathrm{~Pa}$, respectively, and the results are presented in Sec. IV B.

The additional input parameter to be used in FENE-CR model is the finite extensibility parameter $\mathrm{L}$ that has been estimated using Eq. (19). For polystyrene, $\theta=109.5^{\circ}, j=2$, $M_{u}=\mathrm{g} / \mathrm{mol}, M_{w}=110000 \mathrm{~g} / \mathrm{mol}, C_{\infty}=9.6$, and $\nu=0.57$ have been taken corresponding 

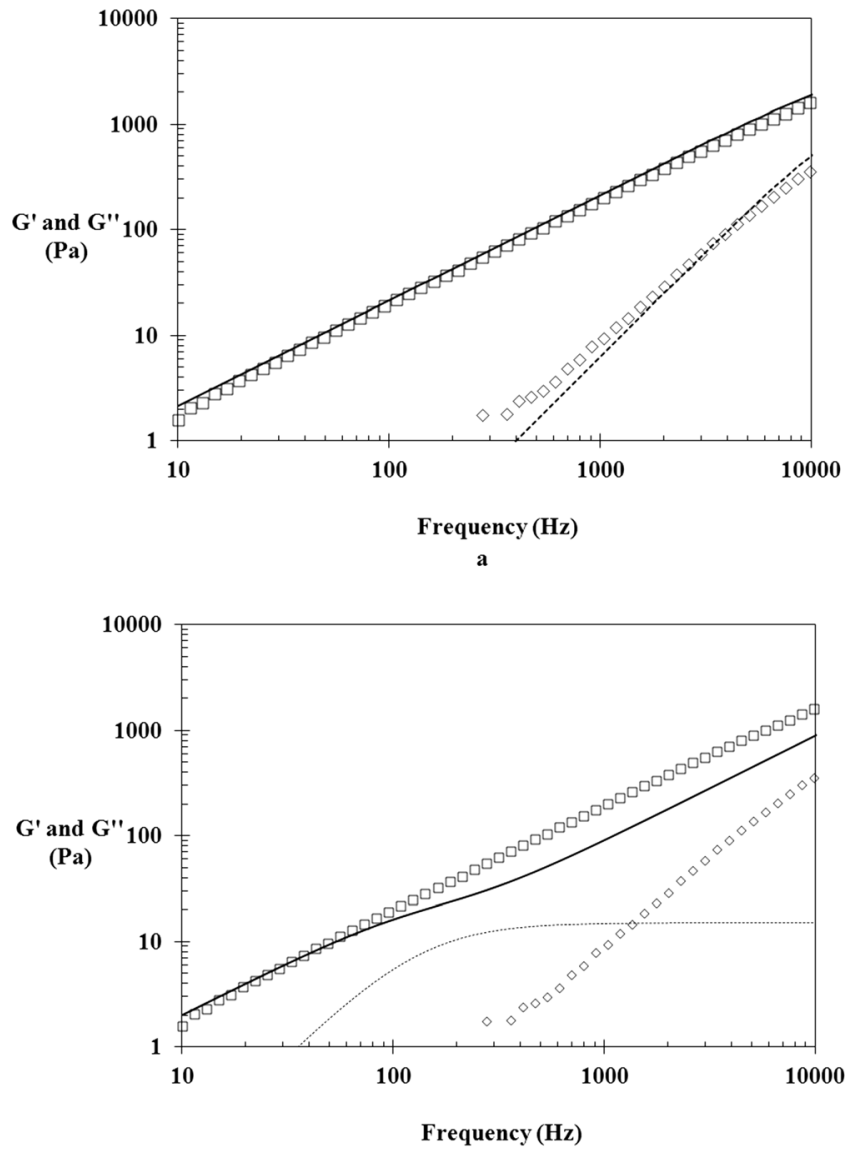

b

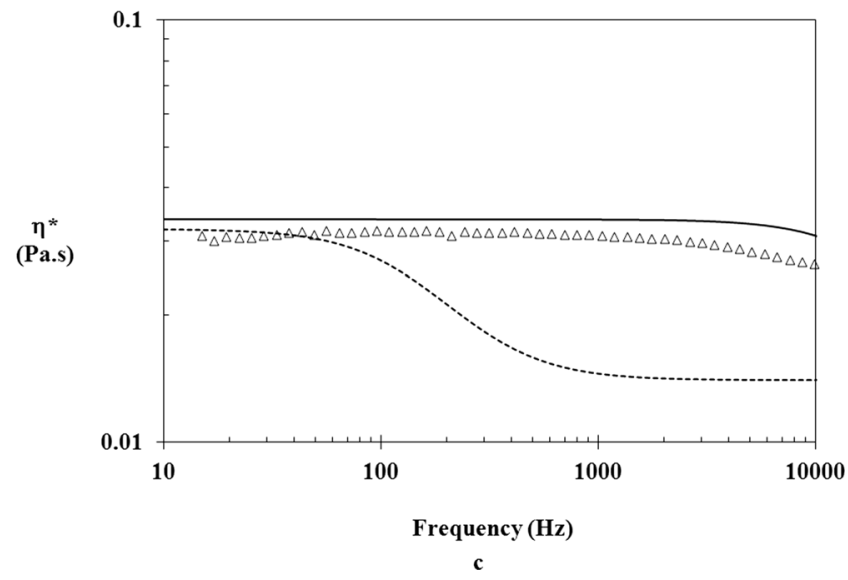

FIG. 8. Plots of $(\diamond)$ storage $G^{\prime},(\square)$ loss modulus $G^{\prime \prime}$ and $(\Delta)$ complex viscosity measured in small amplitude oscillatory shear experiments for the DEP $+2.5 \%$ PS and comparison with the single-mode Maxwell description where the model parameters are derived from two different origins. (a) best fit of experimental PAV data of $\mathrm{G}^{\prime}$ and $\mathrm{G}^{\prime \prime}, \lambda=8 \mu \mathrm{s}$ and $g=2469 \mathrm{~Pa}$; (b) best fitting of $\eta^{*}$ with Maxwell model based on experimentally determined filament thinning data, $\lambda_{\text {ext }}=1.19 \mathrm{~ms}, g=15 \mathrm{~Pa}$. using the experimental filament thinning relaxation time; (c) plot of the experimental complex viscosity $\eta^{*}$ compared with Maxwell model parameters derived in (-) a and (---) b. 
to $L \sim 15$. The value of the Ohnesorge number $\mathrm{Oh}=\eta_{0} / \sqrt{\rho \mathrm{R}_{0} \sigma}(\sim 0.2029)$ justifies accounting for the inertia in the simulation. This is in sharp contrast to some recent work [Olagunju (2011)] where inertia is not considered. Other important characteristics, such as the dimensionless transient extensional viscosity $\eta_{\text {ext }} / \eta_{0}$ (Trouton ratio)

$$
\operatorname{Tr}=\frac{3 \eta_{s} v_{z}+\sigma_{z z}-\sigma_{r r}}{\eta_{0} \dot{\varepsilon}_{\text {mid }}}
$$

where the deformation rate $\dot{\varepsilon}_{\text {mid }}=-\left[2 / D_{\text {mid }}(t)\right]\left[d D_{\text {mid }}(t) / d t\right]$ or the associated Hencky strain accumulated at the midfilament given by $\varepsilon=2 \ln \left(D_{0} / D_{\text {mid }}(t)\right)$ may also be computed quite easily. It is understood that it is Trouton ratio based on the extension rate.

\section{B. Midfilament evolution}

Filament stretching simulations of the non-Newtonian polymer solution $(\mathrm{DEP}+2.5$ wt. \% PS110), for a stretching distance of $0.8 \mathrm{~mm}$ (or aspect ratio $L_{\mathrm{f}} / R_{0}=2.3$ ), using both $\lambda_{\text {ext }}$ and $\lambda_{\text {lve }}$ in Oldroyd-B and FENE-CR constitutive equations have been performed and the midfilament evolution results are compared against experimental measurements (Fig. 9). Experimentally, the midfilament initially thins with time between the piston cessation of motion and $\sim 8.5 \mathrm{~ms}$ indicating a viscous driven thinning mechanism [Bazilevsky et al. (1990); Liang and Mackley (1994); Entov and Hinch (1997); Kolte and Szabo (1999); McKinley (2005)]. At $\sim 8.5 \mathrm{~ms}$, a sudden change occurs with the midfilament following an exponential decay characteristic of an elasto-capillary driven thinning mechanism [Bazilevsky et al. (1990); Renardy (1994); Renardy (1995); Brenner (1996), Bazilevsky et al. (1997); Eggers (1997)].

The simulation performed with the short relaxation time $\lambda_{\text {lve }}(\sim 8 \mu \mathrm{s})$ is presented in Figs. 9(a) and 9(c) for the Oldroyd-B and FENE-CR models, respectively, and both show a relatively good agreement with experiments in the initial decreasing zone of midfilament diameter, regardless of the constitutive model used [Figs. 9(a) and 9(c)]. However, both of the $8 \mu$ s simulations do not capture the long time behavior of the filament decay and show a thinning very similar to the Newtonian case. This result can be anticipated as the very small value of $\lambda$ means that polymer addition mainly contributes to the viscous dissipation as the corresponding Weissenberg number $\lambda_{z} \dot{\varepsilon}$ is of order of 0.015 . This is far too low compared to the critical value of Weissenberg number of 0.5 required to achieve the coil-stretch transition for the polymer [Bazilevsky et al. (1990); Tuladhar and Mackley (2008)]. This argument also indicates that a longer relaxation time of order of $1 \mathrm{~ms}$ is necessary in order to induce polymer chain stretch and subsequently induce elastic thinning effects as the filament thinning strain rate $\dot{\varepsilon}$ has been estimated at $\sim 500 \mathrm{~s}^{-1}$, when the midfilament $\left(D_{\text {mid }} \sim 200 \mu \mathrm{m}\right)$ experimentally shifts from linear decay to exponential decay.

Simulations with both Oldroyd-B and FENE-CR models using the filament thinning relaxation time of $1.19 \mathrm{~ms}(D e=0.47)$ exhibited a closer match to the final observed experimental filament thinning and break-up behavior as shown in Fig. 9(b) for the Oldroyd-B model and Fig. 9(d) for the FENE-CR model. There are few differences between the Oldroyd-B and the FENE-CR at least regarding the mid-diameter evolution, $D_{\text {mid }}(t)$ as shown in Fig. 9. This is expected since the FENE-CR model is equivalent to the Oldroyd-B one when $L$ the extensibility is taken to be infinite. However, when one compares the transient profiles some difference can be drawn as the formation of a small bead in the FENE-CR model which is not present in the Oldroyd-B model, as shown in Fig. 9. In contrast, the filament for the Oldroyd-B model lasts longer. The extensibility $L$ 

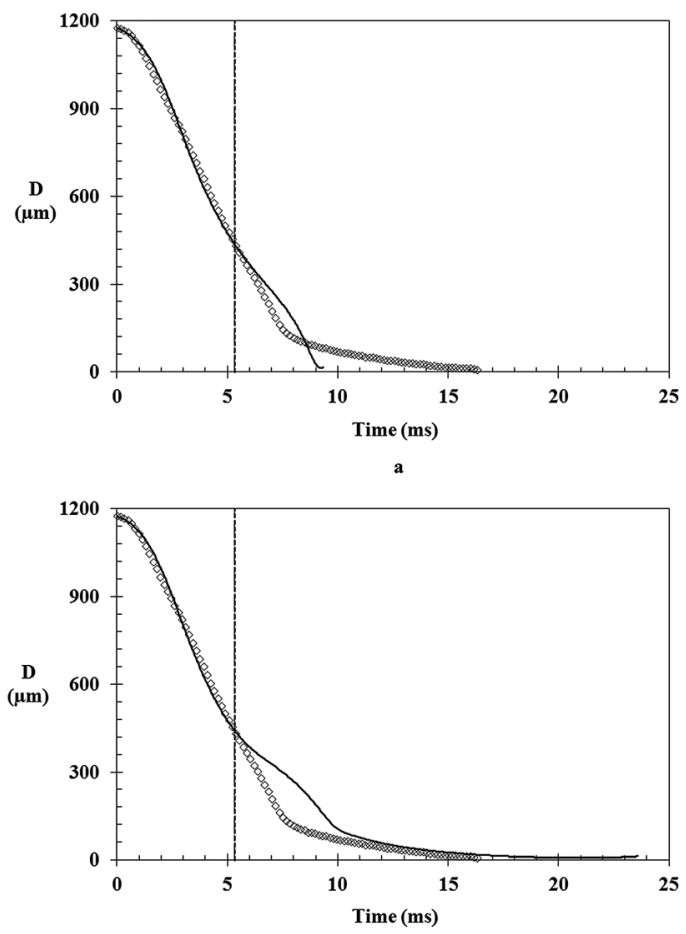

b

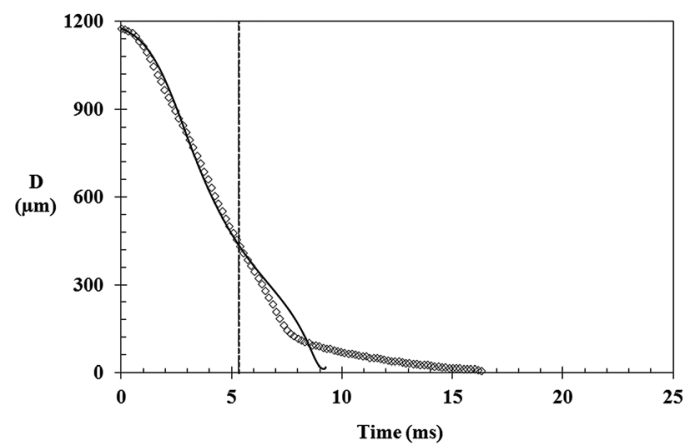

c

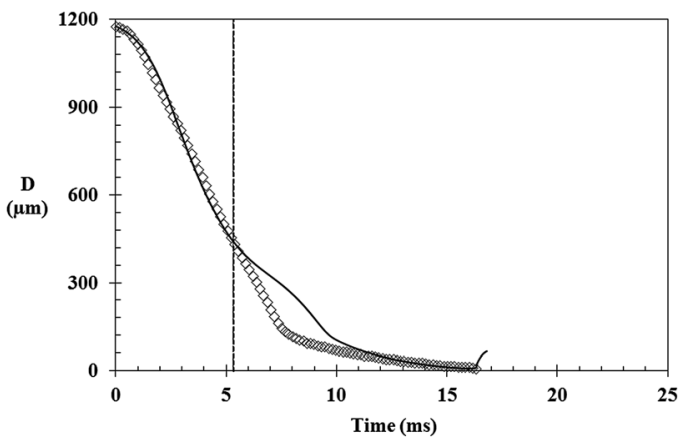

d

FIG. 9. Comparison of $(\diamond)$ experimental midfilament data and (-) simulated midfilament diameters as a function of time. (a) Oldroyd-B, $\lambda=8 \mu \mathrm{s}, g=2469 \mathrm{~Pa}, D e=0.00145$; (b) Oldroyd-B, $\lambda=1.19 \mathrm{~ms}, g=15 \mathrm{~Pa}$, $D e=0.47$; (c) FENE-CR, $\lambda=8 \mu \mathrm{s}, g=2469 \mathrm{~Pa}, D e=0.00145$; (d) FENE-CR $\lambda=1.19 \mathrm{~ms}, g=15 \mathrm{~Pa}$, $D e=0.47$. (--) Represents piston cessation of motion. 
influences the onset of the inertio-viscoelastic balance as well as the filament thinning at longer times, as found in separate simulations performed for a range of $L$ values comprised between 2 and 1500. The infinite extensibility of the Oldroyd-B model did not produce the correct break-up time which was found to be much larger than the experimental results and the FENE-CR results. This supports the choice of the FENE-CR model as a more adequate constitutive equation to address quantitatively the fluid filament stretching and thinning process compared to Oldroyd-B model. The FENE-CR will thus be used for the remaining aspects of the paper.

Even with the FENE-CR model and the chosen filament thinning relaxation time of $1.19 \mathrm{~ms}$, discrepancies were noted compared to the experimental results, particularly in the time window between 6 and $10 \mathrm{~ms}$. Just after the pistons stop, the numerically calculated filament diameter remained larger than the experimental one. It ought to be mentioned that this discrepancy is only observed for a stretching distance of $0.8 \mathrm{~mm}$, as shown in Fig. 9. It is very seldom that such short displacements have been considered in the literature. It may be concluded at this point that an approach based on a single relaxation mode model may not be sufficient to describe properly the full process even for dilute polymer solutions. The discrepancy in relaxation times which are measured, respectively, in the linear and in nonlinear viscoelastic regime is still a matter of discussion in the literature. This discrepancy that other authors have noticed [Wagner et al. (2005); Tirtaatmadja et al. (2006)] may be attributed to the fluid response in the linear regime when using the PAV device, whereas the thinning experiment enables access to the highly nonlinear mode regime. To put it short, the difference between these two time scales could be the manifestation of the multimode nature of the polymeric solution [Ferry (1980)]. A multimode model may be needed to better represent the transition, observed experimentally, from the initial viscous thinning behavior at short time and the elasto-capillary thinning behavior at longer times. It is worth noting that in our approach, the extensibility factor is chosen on physical grounds [see Eq. (19)], and the relaxation times are determined from linear and nonlinear viscoelastic measurements. The fluids which have been fully characterized in the linear viscoelastic region were modeled with a single-mode viscoelastic constitutive equation. This represents a first step, while recognizing that multimode modeling may be necessary, with different timescales in order to deal with the stretching problem. This is in line with some of the conclusions of Wagner et al. (2005).

In the case of filament stretching and thinning of the non-Newtonian fluid for a stretching distance of $1.6 \mathrm{~mm}$ (or aspect ratio $L_{\mathrm{f}} / R_{0}=3.6$ ), a good agreement was also obtained between simulation using FENE-CR model and experiment as shown in Fig. 10. In this simulation, the best fit of the experimental piston motion was again used. This excellent agreement between experiment and simulation for a larger stretching distance highlights the fact that the 1D model is even better as the slenderness approximation is approached. Moreover, since the stretching velocity is low $V_{p} / U_{c}=0.31<1$ (with $U_{c}=R_{0} / \tau$ ), the $1 \mathrm{D}$ model may become remarkably accurate as pointed out by Yildirim and Basaran (2001).

\section{Transient characteristics of the stretching and thinning process}

Comparison of simulated transient profiles with the experimental ones is shown in Fig. 11 for the FENE-CR model. The addition of polymer contributes to stabilize the filament resulting in preventing the early time rupture of the filament and the formation of a central drop. In sharp contrast to the Newtonian fluid, a long lasting uniform filament is observed and this was also numerically well predicted. Figures 10(b) and 11(b) are 3D representations of the filament at the end of the simulation close to break-up and show a 

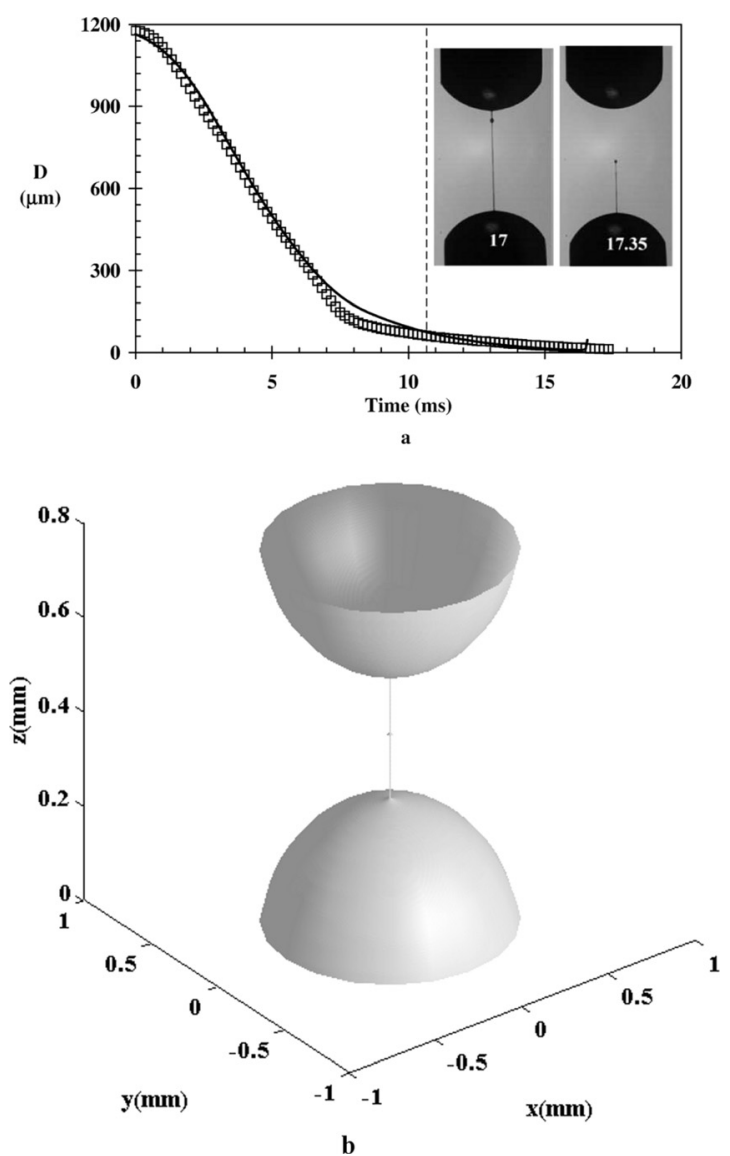

FIG. 10. (a) Comparison of the midfilament evolution of (-) simulation prediction of the FENE-CR model with $D e=0.47$ and $(\square)$ experimental measurements of the DEP +2.5 wt. \% PS. Initial gap size: $0.6 \mathrm{~mm}$, final gap size: $2.2 \mathrm{~mm}$ (or aspect ratio $L_{\mathrm{f}} / R_{0}=3.6$ ), pistons relative velocity: $150 \mathrm{~mm} / \mathrm{s}$. (---) Represents piston cessation of motion. (b) $3 \mathrm{D}$ representation of the filament close to break-up.

nascent or formed beads in the middle of the filament. The formation of beads on string also has been observed experimentally (snap shot of profile taken at $16.8 \mathrm{~ms}$ or $17 \mathrm{~ms}$, respectively, inset in Fig. 10(a) or 11(a) although they did not necessarily form at the same location as predicted in the simulation. This difference in location, asymmetric in the experimental case, may be due to a slight variation in velocity between the upper and the lower pistons or to a none perfectly symmetrical fluid quantity on top and bottom pistons.

Figure 12 presents the evolution of the apparent Trouton ratio, $\eta_{\mathrm{e}, \mathrm{app}} / \eta_{0}$, as a function of the Hencky strain for FENE-CR constitutive model as well as experimental data. Numerically, the apparent Trouton ratio has been estimated using Eq. (29), whereas, experimentally, the midfilament diameter was used to obtain the transient extensional viscosity $\eta_{\text {ext }}$ [Anna and McKinley (2001)]

$$
\eta_{e x t}=-\frac{(2 X-1) \sigma}{d D_{\text {mid }}(t) / d t}
$$

where $X$ is a geometrical factor that corrects for the nonperfectly cylindrical shape of the filament. The apparent extensional viscosity, where $\eta_{\text {e,app }}=\eta_{\text {ext }} /(2 X-1)$, was used 


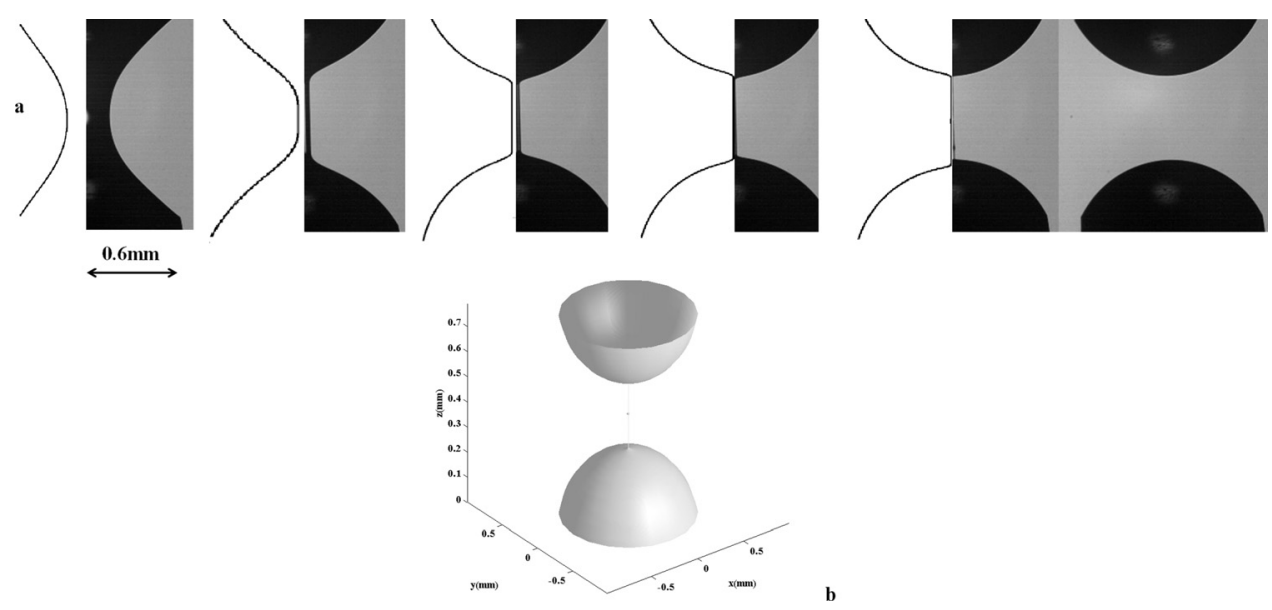

FIG. 11. (a) (left) Photographs of the filament break-up captured with the Cambridge Trimaster (top) and the corresponding numerical profiles (bottom) at different specified times. Initial gap size: $0.6 \mathrm{~mm}$, final gap size: $1.4 \mathrm{~mm}$, pistons relative velocity: $150 \mathrm{~mm} / \mathrm{s}$. (right) the FENE-CR $D e=0.47$ transient profile predictions in the case of DEP + 2.5 wt. \% PS110; (b) 3D representation corresponds to the filament at the end of the simulation. Times are, from left to right: $5.3 \mathrm{~ms}, 10.2 \mathrm{~ms}, 13.5 \mathrm{~ms}, 15.2 \mathrm{~ms}, 16.8 \mathrm{~ms}$, and $17 \mathrm{~ms}$.

preferably to $\eta_{\text {ext }}$ to remove all effects due to the geometrical correction introduced by varying the value of $X$. The first derivative of the midfilament has been obtained by direct differentiation of the midfilament diameter $\left[d D_{\text {mid }}(t) / d t \approx D_{\text {mid }}\left(t_{i}\right)-D_{\text {mid }}\left(t_{i-1}\right) / t_{i}-t_{i-1}\right]$, after a smoothing operation that consisted in performing a running average of window size of seven points.

It is worth noting that the experimental device by itself is unique in its ability to access high filament stretching strain rate which subsequently enables probing extensional rheology of low viscosity weakly viscoelastic fluids with extensional relaxation times as short as $200 \mu$ s [Vadillo et al. (2010a); Ardekani et al. (2010); Campo-Deaño and Clasen (2010)]. This experimental capability can bring new insight in the understanding of the fluid microstructure for different flow conditions as well as providing experimental data to an area of rheology limited, until now, to simulations. As stated by Ardekani et al. (2010), the experimental determination of extensional viscoelastic properties for these very low viscosity and weakly elastic liquids is a particular challenge using traditional rheometers.

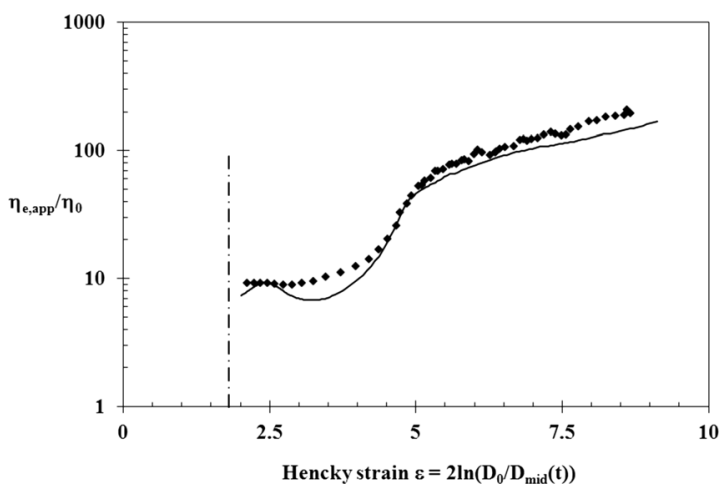

FIG. 12. The simulated apparent Trouton ratio as a function of the Hencky strain for (-) the FENE-CR model with $D e=0.47$ (aspect ratio of 2.3) and $(\diamond)$ experimental results [Eq. (30) with $X=1$ ]. (--) Represents piston cessation of motion. 


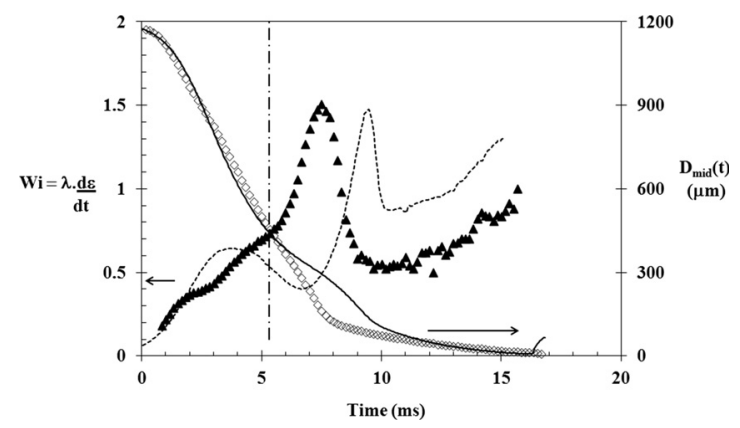

FIG. 13. Comparison of Weissenberg number for long time relaxation time ( $1.19 \mathrm{~ms})$ calculated for $(\boldsymbol{\Delta})$ experimental and (---) numerical FENE-CR constitutive model. $(\diamond)$ experimental midfilament data and $(-)$ simulated midfilament diameters as a function of time. Constitutive model: FENE-CR $\lambda=1.19 \mathrm{~ms}, g=15 \mathrm{~Pa}, D e=0.47$. (--) Represents piston cessation of motion.

A reasonable agreement can be observed between the simulated and the experimental apparent Trouton ratio. FENE-CR constitutive model was capable of capturing correctly, the complex behavior of the extensional viscosity with the Hencky strain in particular in the region of large deformation. The discrepancy noticeable at low Hencky strain may be attributed to experimental measurement errors; the use of approximate piston motions the limitation of the constitutive model leading to difficulty to reproduce the transition between visco-capillary thinning to elasto-capillary thinning. Figure 13 presents the Weissenberg number (defined as $W i=\lambda . \dot{\varepsilon}$ ) as a function of time and illustrates critically that the transition between a Newtonian filament decay and a viscoelastic exponential decay occurs in the transition region of $W i$ between 0.5 and 1.0. The figure also shows an apparent mismatch in time delay between the simulated and the experimental curves, which appears to be related to the simulation incorrectly predicting the exact form of decay in the transition region.

Concerning the formation of the bead referred to in Figs. 10 and 11, it may attributed to the inversion in the axial velocity (Fig. 14) that occurs at the stagnation point located at the midposition of the filament $(z=0)$. The upper right hand diagram shows, in particular, the velocity direction inside the filament during the stretching process and clearly pinpoints a change of direction (see lower right hand diagram). This inversion leads to fluid build up in the center of the filament, whereas the ends of the filament thin and pinch-off occur near the pistons (Fig. 6) for a Newtonian fluid. In the viscoelastic case, the pinching at the pistons is delayed due to the growth of the elastic stress of the strain-hardening filament which overcomes the surface tension forces, corresponding to the main destabilizing forces in the filament. It is also noted that, since the filament is relatively slender, the deformation becomes close (around $\left.t / t_{\mathrm{p}}=0.8\right)$ to an uniaxial extension $(\mathrm{V} \sim \mathrm{z})$ before break-up occurs [Figs. 14(b) and 14(c)]. The curves for the axial velocity are quite smooth at the beginning of the stretching but progressively become more and more abrupt with a sharp fall at the center of the filament leading to localized formation of beads. Since modeling has been performed for two different aspect ratios (2.3 and 3.6), it may be noted that in addition of inertia, as stated in Bhat et al. (2010), operating conditions (piston displacement history) may also play a major role in the formation of the bead on a string structure especially when dealing with filament stretching experiment. The bead formation is more prominent in the case of a stretching distance of $1.6 \mathrm{~mm}$ (aspect ratio 3.6) compared to the one at $0.8 \mathrm{~mm}$ (aspect ratio 2.6) as highlighted in Figs. 14(b) and 14(c).

It may be useful to emphasize that the approach proposed in this paper is fully based on theoretical arguments and experimental measurements to determine all the inputs 

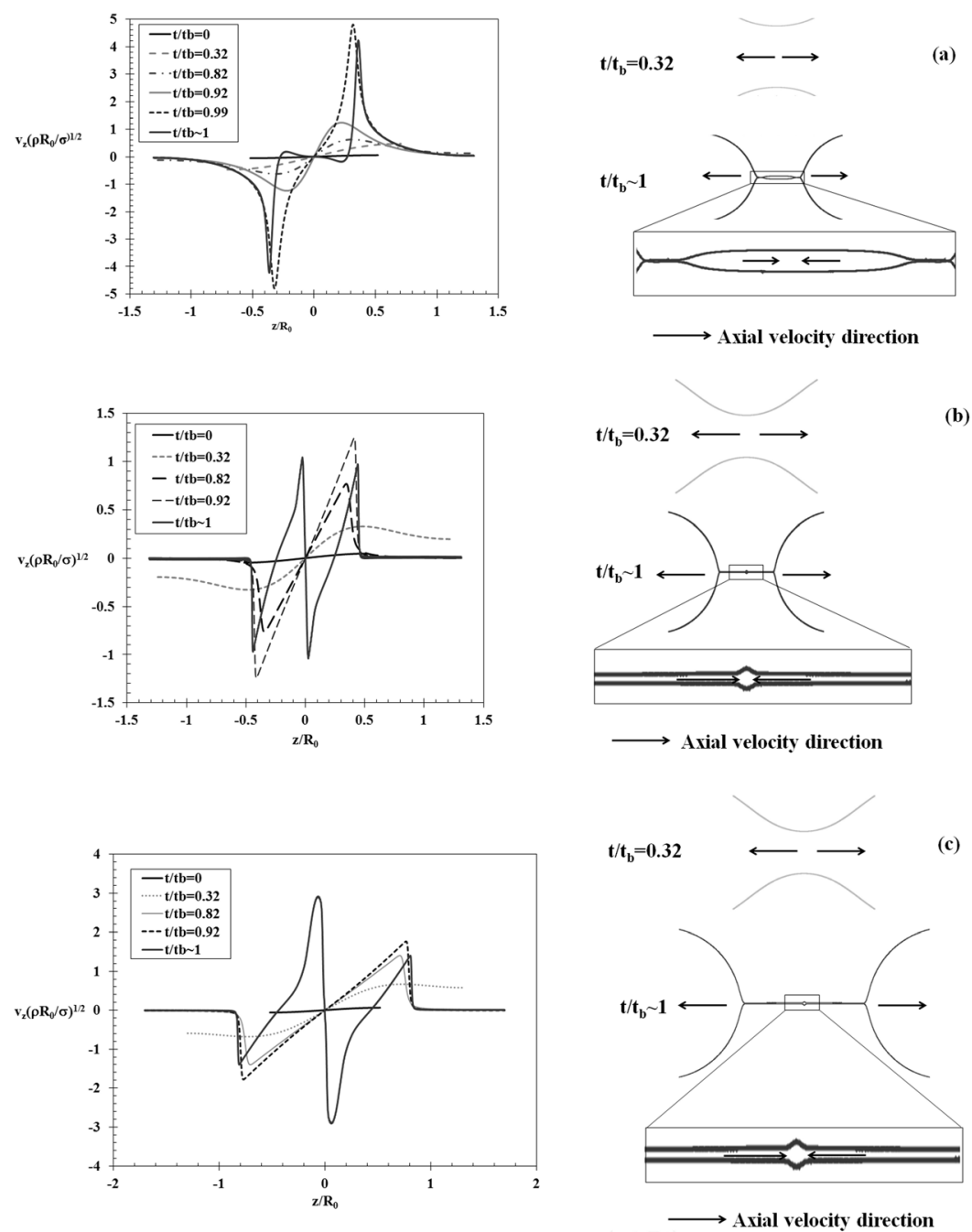

FIG. 14. The computed axial velocity evolution for a stretching distance of $0.8 \mathrm{~mm}$ (aspect ratio 2.3) in (a) Newtonian case and (b) the FENE-CR model with $D e=0.47$. (c) shows the evolution for a stretching distance of $1.6 \mathrm{~mm}$ (or aspect ratio $L_{\mathrm{f}} / R_{0}=3.6$ ) and FENE-CR model with $D e=0.47$; time is scaled by $t_{\mathrm{b}}$, the pinch-off time. The right hand diagram shows the velocity direction inside the filament during the stretching.

parameters of the model such as the extensibility and the relaxation's times in the linear and the nonlinear regimes. This work highlights the need to fully employ the piston displacement history and the necessity of a multimode approach. As far as, we are aware off, there is no quantitative comparison in the literature between the experiments and simulations both in terms of midfilament evolution and transient profile for stretching experiments with low viscosity and weakly viscoelastic fluids.

\section{v. CONCLUSIONS}

In this paper, a complete modeling of the fluid filament stretching and thinning processes has been performed using a one-dimensional approach for both Newtonian and non-Newtonian fluids. The approach takes proper account of the initial piston filament 
stretching motion using an ALE formulation and this was found to be an important factor that needs to be considered to obtain a good matching with experiment. The constitutive equations considered for non-Newtonian fluids was the well known Oldroyd-B and FENE-CR formulated in the 1D approximation. The simulations were tested against results obtained from the Cambridge Trimaster apparatus, and quantitative comparisons between the experimental and numerical results were performed for the piston deformation and subsequent transient surface tension driven thinning phenomena. The behavior of the Newtonian fluid was very well predicted by the numerical model. In the case of the non-Newtonian fluid, the single-mode Oldroyd-B model shows some discrepancies at long times probably related to the infinite extensibility of this model, while the singlemode FENE-CR gave better results at least in terms of break-up time even, if it fails to accurately describe the full elongational transient profile. In order to achieve a good fit to the experimental data, it was necessary to use the relaxation time measured from experimental filament thinning. The relaxation time derived from the PAV was far too short to have an observable effect. It is concluded that either the relaxation time of the polymer increases during stretch or a single-mode description of the LVE is inadequate, and longer modes exist but are not detected by the PAV. In order to capture the full profile and predict the correct break-up time with self consistent results to the PAV, it is suspected that a mono modal constitutive equation is insufficient and that a multimodal approach will need to be explored. The extreme sensitivity of the simulation to the pistons velocity profile must also be taken into account and this factor may also influence the discrepancy in the transition region before the onset of the exponential thinning behavior. Finally, it may be necessary to modify the constitutive equation or consider other closure equations to reflect a change in relaxation with polymer stretch. The 1D model for the many reasons mentioned in this paper may prove to be convenient in order to explore other complex situations either in terms of constitutive equations and/or in terms of operating conditions.

\section{ACKNOWLEDGMENTS}

M.T. and A.S. wish to acknowledge financial support from ANR PAN'H 2008 CATIMINHY project. M.T. thanks Jens Eggers for discussions. D.V. wishes to thank the Engineering and Physical Sciences Research Council (UK) and industrial partners in the Innovation in Industrial Inkjet Technology project, EP/H018913/1 for financial support.

\section{References}

Ambravaneswaran, B., E. D. Wilkes, and O. A. Basaran, "Drop formation from a capillary tube: Comparison of one-dimensional and two dimensional analyses and occurrence of satellite drops," Phys. Fluids 14, 2606 (2002).

Anna, S. L., and G. H. McKinley, "Elasto-capillary thinning and breakup of model elastic liquids," J. Rheol. 45, 115-138 (2001).

Ardekani, A. M., V. Sharma, and G. H. McKinley, "Dynamics of bead formation, filament thinning and breakup in weakly viscoelastic jets," J. Fluid Mech. 665, 46-56 (2010).

Bach, A., H. K. Rasmussen, P-Y. Longin, and O. Hassager, "Growth of non-axisymmetric disturbances of the free surface in the filament stretching rheometer: Experiments and simulation," J. Non-Newtonian Fluid Mech. 180, 163-186 (2002).

Bazilevsky, A. V., V. M. Entov, and A. N. Rozhkov, "Liquid filament microrheometer and some of its applications," in Third European Rheology Conference, edited by D. R. Oliver (Elsevier Applied Science, Amsterdam, Netherlands, 1990), pp. 41-43.

Bazilevsky, A. V., V. M. Entov, and A. N. Rozhkov, "Failure of polymer solutions filaments," Polym. Sci., Ser. B 39, 316-324 (1997). 
Bhat, P. P., S. Appathurai, M. T. Harris, M. Pasquali, G. H. McKinley, and O. A. Basaran, "Formation of beadson-a-string structures during break-up of viscoelastic filaments,” Nat. Phys. 6, 625-631 (2010).

Brenner, M. P., J. R. Lister, and H. A. Stone, "Pinching threads, singularities and the number 0.0304," Phys. Fluids 8, 2827-2836 (1996).

Campo-Deaño, L., and C. Clasen, "The slow retraction method (SRM) for the determination of ultra-short relaxation times in capillary breakup extensional rheometry experiments," J. Non-Newtonian Fluid Mech. 165, 1688-1699 (2010).

Castrejon-Pita, J. R., N. F. Morrison, O. G. Harlen, G. D. Martin, and I. M. Hutchings, "Experiments and Lagrangian simulations on the formation of droplets in continuous mode," Phys. Rev. E 83, 016301 (2011).

Chilcott, M. D., and J. M. Rallison, "Creeping flow of dilute polymer solutions past cylinders and spheres," J. Non-Newtonian Fluid Mech. 29, 381-432 (1988).

Clasen, C., J. Eggers, M. A. Fontelos, J. Li, and G. H. McKinley, "The beads-on-string structure of viscoelastic threads," J. Fluid Mech. 556, 283 (2006a).

Clasen, C., J. P. Plog, W.-M. Kulicke, M. Owens, C. Macosko, L. E. Scriven, M. Verani, and G. H. Mckinley, "How dilute are dilute solutions in extensional flows?," J. Rheol. 50(6), 849-881 (2006b).

Crassous, J., R. Régisser, M. Ballauff, and N. Willenbacher, "Characterisation of the viscoelastic behaviour of complex fluids using the piezoelastic axial vibrator," J. Rheol. 49, 851-863 (2005).

Dong, H., W. W. Carr, and J. F. Morris, "An experimental study of drop-on-demand drop formation," Phys. Fluids 18, 072102 (2006).

Eggers, J., and T. F. Dupont, "Drop formation in a one-dimensional approximation of the Navier-Stokes equation,” J. Fluid Mech. 262, 205 (1994).

Eggers, J., "Nonlinear dynamics and breakup of free-surface flows," Rev. Mod. Phys. 69, 865-929 (1997).

Entov, V. M., and E. J. Hinch, "Effect of a spectrum relaxation times on the capillary thinning of a filament elastic liquids," J. Non-Newtonian Fluid Mech. 72, 31-53 (1997).

Ferry, J. D., Viscoelastic Properties of Polymers (John Wiley \& Sons Inc., New York, 1980).

Fontelos, M. A., and J. Li, "On the evolution and rupture of filaments in Giesekus and FENE models," J. NonNewtonian Fluid Mech. 118, 1-16 (2004).

Furlani, E. P., and M. S. Hanchak, "Nonlinear analysis of the deformation and breakup of viscous microjets using the method of lines,” Int. J. Numer. Methods Fluids 65(5), 563-577 (2011).

Graessley, W. W., "Polymer chain dimensions and the dependence of viscoelastic properties on the concentration, molecular weight and solvent power," Polymer 21, 258-262 (1980).

Groß, T., L. Kirschenmann, and W. Pechhold, "Piezo axial vibrator (PAV)—A new oscillating squeeze flow rheometer," in Proceedings Eurheo, edited by H. Munsted, J. Kaschta, and A. Merten (Erlangen, Germany, 2002).

Hanchak, M. S. "One dimensional model of thermo-capillary driven liquid jet break-up with drop merging," Ph.D. thesis, University of Dayton, Dayton, OH (2009).

Hoath, S. D., G. D. Martin, T. R. Tuladhar, M. R. Mackley, I. M. Hutchings, and D. C. Vadillo, "Link between ink rheology, drop-on-demand jet formation and printability,” J. Imaging Sci. Technol. 53(4), 041208 (2009).

Hoeve, W. V., S. Gekle, J. H. Snoeijer, M. Versluis, M. P. Brenner, and D. Lohse, "Breakup of diminutive Rayleigh jets," Phys. Fluids 22, 122003 (2010).

Kirschenmann, L., Ph.D. thesis, Institut fur dynamische materialprunfung (IdM), University of Ulm, Ulm, Germany (2003).

Kolte, M. I., and P. Szabo, "Capillary thinning of polymeric filaments," J. Rheol. 43, 609-625 (1999).

Kuroiwa, T., N. Ishikawa, D. Obara, F. Vinet, E. S. Ang, A. Guelbi, and A. Soucémarianadin, "Dispensing of polymeric fluids for BIO-MEMS applications," Proceedings of the IS \& T's Nineteenth International Congress on Advances in Non-Impact Printing Technologies, New Orleans, October 2003.

Liang, R. F., and M. R. Mackley, "Rheological characterization of the time and strain dependence for polyisobutylene solutions," J. Non-Newtonian Fluid Mech. 52, 387-405 (1994).

Ma, W. K. A., F. Chinesta, T. Tuladhar, and M. R. Mackley, "Filament stretching of carbon nano tube suspension," Rheol. Acta 47, 447-457 (2008).

Matta, J. E., and R. P. Tytus, "Liquid stretching using falling cylinder," J. Non-Newtonian Fluid Mech. 35, 215-229 (1990). 
Matallah, H., K. S. Sujatha, M. J. Banaai, and M. F. Webster, "Single and multi-mode modelling for filament stretching flows," J. Non-Newtonian Fluid Mech. 146, 92-113 (2007).

McKinley G. H., and T. Sridhar, "Filament stretching rheometry of complex fluids," Annu. Rev. Fluid Mech. 34, 375-415 (2002).

McKinley, G. H., "Visco-elastic-capillary thinning and break-up of complex fluid," Br. Soc. Rheol. 1-49 (2005), Rheology Reviews 2005.

Morrison, N. F. and O. G. Harlen, "Viscoelasticity in inkjet printing," Rheol. Acta 49, 619-632 (2010).

Nielsen, J. K., H. K. Rasmussen, O. Hassager, and G. H. McKingley, "Elongation viscosity of monodisperse and bidisperse polystyrene melts," J. Rheol. 50(4), 453-476 (2006).

Olagunju, D. O., "Extensional flow of a viscoelastic filament governed by the FENE-CR model," Int. J. NonLinear Mech. 46(1), 73-78 (2011).

Orr, N. V., and T. Sridhar, "Probing the dynamics of polymer solutions in extensional flow using step strain rate experiments," J. Non-Newtonian Fluid Mech. 82, 203-232 (1996).

Papageorgiou, D. T., “Analytical description of the breakup of liquid jets,” J. Fluid Mech. 301, 109 (1995).

Renardy, M., "Some comments on the surface tension driven breakup (or the lack of it) of the viscoelastic jets," J. Non-Newtonian Fluid Mech. 51, 97-107 (1994).

Renardy, M., "A numerical study of the asymptotic evolution and breakup of Newtonian and viscoelastic jets," J. Non-Newtonian Fluid Mech. 59, 267-282 (1995).

Rodd, L. E., T. P. Scott, J. J. Cooper-White, and G. H. McKinley, "Capillary breakup rheometry of lowviscosity elastic fluids," Appl. Rheol. 15(1), 12-27 (2005).

Tirtaatmadja, V., G. H. McKinley, and J. J. Cooper-White, "Drop formation and breakup of low-viscosity elastic fluids: Effects of molecular weight and concentration,” Phys. Fluids 18, 043101 (2006).

Tuladhar, T. R., and M. R. Mackley, "Filament stretching rheometry and break-up behaviour of low viscosity polymer solutions and ink jets fluids," J. Non-Newtonian Fluid Mech. 148, 97-108 (2008).

Vadillo, D. C., T. R. Tuladhar, A. C. Mulji, S. Jung, S. D. Hoath, and M. R. Mackley, "Evaluation of the inkjet fluid's performance using the 'Cambridge Trimaster' filament stretch and break-up device," J. Rheol. 54(2), 261-282 (2010a).

Vadillo, D. C., T. R. Tuladhar, A. Mulji, and M. R. Mackley, "The rheological characterisation of linear viscoelasticity for ink jet fluids using a piezo axial vibrator (PAV) and torsion resonator (TR) rheometers," J. Rheol. 54(4), 781-799 (2010b).

Yao, M., and G. H. McKinley, "Numerical simulation of extensional deformations of viscoelastic liquid bridges in filament stretching devices," J. Non-Newtonian Fluid Mech. 74, 47-88 (1998).

Yildirim, O. E., and O. A. Basaran, "Deformation and breakup of stretching bridges of Newtonian and shearthinning liquids: Comparison of one- and two-dimensional models," Chem. Eng. Sci. 56(1), 211-233 (2001).

Webster, M. F., H. Matallah, K. S. Sujatha, and M. J. Banaai, "Numerical modelling of step-strain for stretched filaments," J. Non-Newtonian Fluid Mech. 151, 38-58 (2008).

Wagner, C., Y. Amarouchene, D. Bonn, and J. Eggers, "Droplet detachment and satellite bead formation in viscoelastic fluids,” Phys. Rev. Lett. 95, 164504 (2005). 